\title{
Cardiopulmonary factors affecting 6-min walk distance in patients with idiopathic inflammatory myopathies
}

\author{
Naoki Mugii ${ }^{1} \cdot$ Fujiko Someya ${ }^{2}$ (i) \\ Received: 19 April 2018 / Accepted: 10 May 2018 / Published online: 14 May 2018 \\ (C) The Author(s) 2018
}

\begin{abstract}
Idiopathic inflammatory myopathies involve skeletal muscles and can be associated with interstitial lung disease and/or heart dysfunction, which may reduce exercise capacity. We aimed to clarify cardiopulmonary factors affecting the 6-min walk distance in patients who were able to walk without leg pain or fatigue. Twenty-three patients with inactive adult idiopathic inflammatory myopathies, and 18 age- and gender-matched healthy controls were evaluated for hemodynamic responses using noninvasive impedance cardiography during the 6-min walk test. The patients were also examined by the pulmonary function test for forced vital capacity and diffusing capacity for carbon monoxide (DLCO), and by echocardiography for left ventricular ejection fraction and right ventricular systolic pressure. Interstitial lung disease was diagnosed in 19 patients using high-resolution computed tomography. There was no difference in 6-min walk distance or cardiac output after walking between the patients and healthy controls. However, stroke volume during the 6-min walk test was significantly lower in the patients than in healthy controls, suggesting malfunction in the heart. Moreover, the increased heart rate matched the cardiac output. Spearman's correlation analysis demonstrated a correlation between 6-min walk distance and stroke volume, cardiac output after walking and DLCO, but not left ventricular ejection fraction or right ventricular systolic pressure, as this study lacked the patients with pulmonary hypertension. In conclusion, impaired DLCO due to interstitial lung disease was suggested to be a fundamental parameter affecting exercise capacity, in addition to heart involvement, in patients with idiopathic inflammatory myopathies.
\end{abstract}

Keywords Polymyositis $\cdot$ Dermatomyositis $\cdot$ Exercise capacity $\cdot$ Pulmonary function test $\cdot$ Hemodynamic response $\cdot$ Interstitial lung disease

\section{Introduction}

Interstitial lung disease is a common extra-muscular complication of idiopathic inflammatory myopathies, and lowdiffusing capacity for carbon monoxide (DLCO) is known to increase the risk of mortality [1,2]. A recent study demonstrated that recovery of skeletal muscle strength and reduced creatine kinase levels by medication for idiopathic inflammatory myopathies may improve forced vital capacity (FVC)

Fujiko Someya

fujiko@mhs.mp.kanazawa-u.ac.jp

Naoki Mugii

mugii@med.kanazawa-u.ac.jp

1 Division of Health Science, Kanazawa University Graduate School of Medical Science, Kanazawa, Japan

2 School of Health Sciences, Kanazawa University, Kodatsuno 5-11-80, Kanazawa 920-0942, Japan due to muscular strength reinforcement; however, DLCO and interstitial infiltrates on CT were not ameliorated in most cases [3]. In general, distance during the 6-min walk test (6MWT) correlates with FVC and DLCO in patients with idiopathic pulmonary fibrosis [4]. Therefore, we hypothesized that accompanying interstitial lung disease may be a factor affecting exercise capacity in patients with idiopathic inflammatory myopathies, and examined which pulmonary function parameters affect exercise capacity.

Heart involvement in idiopathic inflammatory myopathies was also detected by cardiac magnetic resonance tomography [5] or echocardiography [6], and these findings may lead to therapeutic treatment for the cause of morbidity [7]. An increased risk of myocardial infarction or conduction abnormalities was reported in patients with idiopathic inflammatory myopathies [8]. Moreover, left ventricular diastolic dysfunction [6] and reduced left ventricular ejection fractions (EF) [5] were observed in patients regardless of the presence 
of myalgia, paresis, and exhaustion. Few studies have demonstrated the relationship between cardiac functions and exercise capacity in patients with idiopathic inflammatory myopathies. It may be difficult to clarify cardiac parameters likely associated with exercise capacity using conventional echocardiography; however, when cardiac impairment was assessed by global longitudinal strain measurement, subclinical heart disease was found in up to $50 \%$ of patients [9].

Referring to other estimates for cardiac parameters, recent studies have demonstrated that impedance cardiography with real-time monitoring can noninvasively evaluate hemodynamic responses during the 6MWT $[10,11]$. Pulmonary hypertension patients with a normal ejection fraction had a lower stroke volume (SV) and cardiac index (CI) compared with healthy controls during walking [10], and patients with chronic obstructive disease exhibited slower responses to increasing cardiac output (CO) [11]. Moreover, the maximal $\mathrm{CO}$ in patients with pulmonary hypertension was observed during the 6MWT by the inert gas rebreathing method [12]. Thus, these noninvasive methods may be available for evaluation of hemodynamic responses during walking to examine the relation between exercise capacity and heart involvement in patients with idiopathic inflammatory myopathies.

In the present study, we investigated lung and cardiac function parameters related to limited exercise capacity in patients with idiopathic inflammatory myopathies. For cardiac function, we compared measurements obtained from patients and age- and gender-matched healthy controls during the 6MWT using noninvasive impedance cardiography.

\section{Methods}

Patients receiving medication for idiopathic inflammatory myopathies were recruited among patients referred to the Rehabilitation Division of Kanazawa University Hospital between November 2014 and September 2017 for consultation on further treatments followed by treatment with glucocorticoids at the acute phase. They received muscular training, pulmonary rehabilitation, and education about activities of daily living. Exclusion criteria for this study were juvenile dermatomyositis, overlap with other connective tissue diseases, muscle weakness in the extremities of less than 4 on manual muscle testing, leg discomfort or pain during the 6MWT, and active myositis, i.e., period of increased serum creatine kinase. In total, 23 patients were assigned to this study (Table 1). According to the criteria of Bohan and Peter or Sontheimer's criteria, 12 of them were diagnosed as dermatomyositis, 10 as clinically amyopathic dermatomyositis, and 1 as polymyositis. On autoantibody analysis, seven had anti-ARS (anti-Jo-1, PL-7, EJ, and OJ), ten had anti-MDA5, two had anti-SRP, three had anti-TIF1- $\gamma$, and
Table 1 Characteristics of subjects

\begin{tabular}{llll}
\hline & Healthy controls $(n=18)$ & Myopathy patients $(n=23)$ & $p$ \\
\hline Gender $(\mathrm{f} / \mathrm{m})$ & $12 / 6$ & $13 / 10$ & 0.51 \\
Age $($ years $)$ & $62(21 ; 74)$ & $60(27 ; 80)$ & 0.81 \\
Height $(\mathrm{cm})$ & $160(147 ; 179)$ & $159(152 ; 177)$ & 0.91 \\
Weight $(\mathrm{kg})$ & $57(37 ; 73)$ & $57(41 ; 72)$ & 0.74 \\
Body mass index & $22(17 ; 25)$ & $21(16 ; 28)$ & 0.54 \\
6MWT distance (m) & $530(430 ; 743)$ & $516(261 ; 669)$ & 0.28 \\
FVC $(\%$ prediction) & - & $97.1(61.3 ; 138.3)$ & \\
DLCO $(\%$ prediction) & - & $60.8(37.8 ; 88.1)$ & \\
EF $(\%)$ & - & $70(44 ; 79)$ & 0.002 \\
RVSP (mmHg) & - & $26(12 ; 35)$ & 0.04 \\
At rest & & & 0.03 \\
Stroke volume (mL) & $69.7(51.3 ; 95.5)$ & $57.6(20.7 ; 92.2)$ & 0.02 \\
Heart rate (beats/min) & $75(57 ; 105)$ & $85(64 ; 117)$ & \\
Cardiac output $(\mathrm{L})$ & $5.5(3.5 ; 7.7)$ & $4.6(1.7 ; 8.0)$ & 0.002 \\
Cardiac index & $3.7(1.9 ; 4.5)$ & $2.7(1.2 ; 4.9)$ & 0.18 \\
At 6 min of 6MWT & & & 0.04 \\
Stroke volume (mL) & $105.8(80.1 ; 136.4)$ & $76.6(16.5 ; 122.0)$ & 0.04 \\
Heart rate (beats/min) & $118(84 ; 161)$ & $134(89 ; 197)$ & \\
Cardiac output (L) & $12.2(8.2 ; 17.1)$ & $9.8(1.5 ; 20.1)$ & \\
Cardiac index & $7.8(5.2 ; 10.6)$ & $6.2(1.1 ; 12.6)$ & \\
\hline
\end{tabular}

Values are median (min; max). FVC, DLCO, EF, and RVSP were not measured in healthy controls 6MWT 6-min walk test, $F V C$ forced vital capacity, $D L C O$ diffusing capacity for carbon monoxide, $E F$ ejection fraction, RVSP right ventricular systolic pressure 
one was unknown. Interstitial lung disease was diagnosed in 19 patients by specialists of respiratory medicine using high-resolution computed tomography, mainly with the presence of ground-glass opacities. The study was approved by the human ethics committee of Kanazawa University, conforming to the provisions of the Declaration of Helsinki. Written informed consent for the study was obtained from all patients and 18 healthy controls before the performance of the 6MWT.

Hemodynamic responses during the 6MWT were measured using the PhysioFlow Q-Link (Manatec Biomedical, France), which weighed $200 \mathrm{~g}$ and was wearable. Six disposable electrodes (BlueSensor SP, Ambu, Denmark) connecting to the equipment were placed around the chest for impedance cardiography, as previously described [10]. The 6MWT was performed following the Guidelines of the American Thoracic Society in a 30-m corridor [13]. SV, heart rate, $\mathrm{CO}$, and $\mathrm{CI}$ were recorded at rest for autocalibration for $30 \mathrm{~s}$ before the 6MWT. Subsequently, the data were automatically averaged every $10 \mathrm{~s}$ during the $6 \mathrm{MWT}$, and values at 6 min of walking were collected for analyses (Fig. 1).

The clinical data of percentages of predicted FVC and DLCO (FVC\% and DLCO\%) from pulmonary function tests, and left ventricular EF and right ventricular systolic pressure (RVSP) from echocardiography were collected by reviewing the medical records of the patients. These evaluations were not performed for healthy controls, as we expected the values to be normal.

\section{Statistics}

Clinical characteristics were compared between the patients and healthy controls using the Mann-Whitney test for numerical data, and chi-square test for gender distribution. The relation between SV at rest and that at 6 min was examined using Spearman's correlation coefficients for all subjects. Correlation analyses were performed for 6MWT distance and $\mathrm{FVC} \%$, DLCO\%, EF, RVSP, SV, and $\mathrm{CO}$ at rest and at 6 min. JMP 11.0 (SAS Institute Inc., Cary, NC) was used for statistical analysis. A $p$ value of $<0.05$ was considered significant.

\section{Results}

There was no difference in gender distribution, age, or anthropometric measurements between patients and healthy controls. The median DLCO $\%$ was low at $60.8 \%$ in patients, and seemed to be poorer than the median $\mathrm{FVC} \%$, which was $97.1 \%$ (Table 1). The EF in patients was not impaired at $70 \%$, except in two patients showing $\mathrm{EF}<60 \%$. RVSP reflects pulmonary
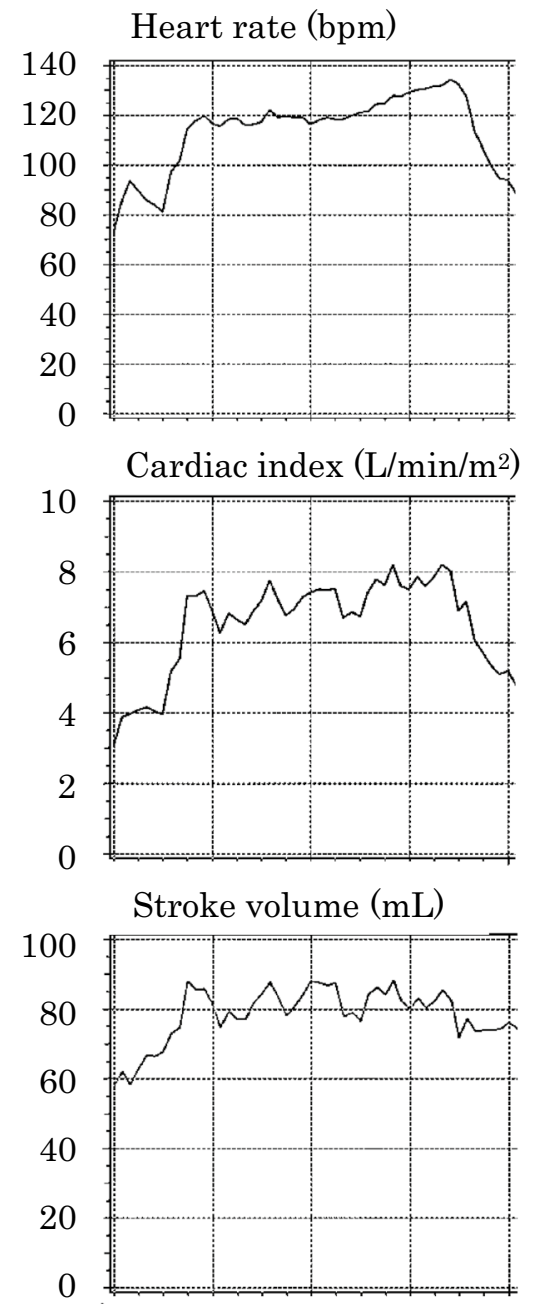

Cardiac output (L/min)

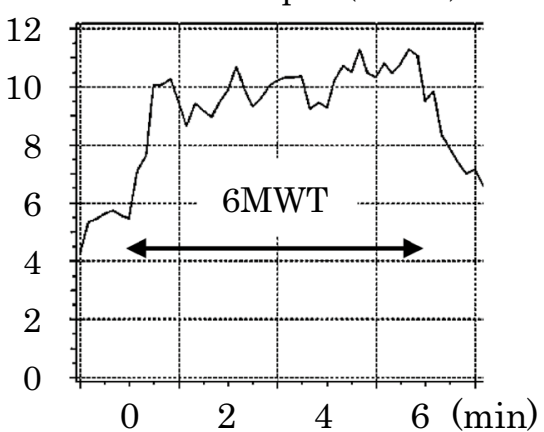

Fig. 1 Hemodynamic responses recorded by noninvasive impedance cardiography during the 6-min walk test (6MWT) in a patient

artery systolic pressure and $>$ approximately $35 \mathrm{mmHg}$ indicates pulmonary hypertension [14], and there were no patients with high RVSP in this study. The SV, CO, and CI at rest and at 6 min were significantly lower in patients than in healthy controls. Patient heart rates were high, but there was no significant difference between patients and healthy controls at $6 \mathrm{~min}$. The $6 \mathrm{MWT}$ distance was shorter for patients, but the difference 


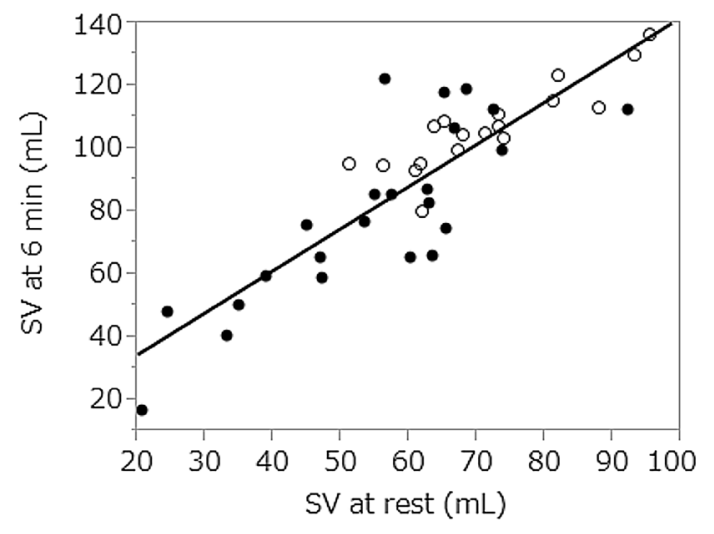

Fig. 2 Relationship between stroke volume (SV) at rest and at 6 min during the 6-min walk test. Open circles represent healthy controls and closed circles represent patients with idiopathic inflammatory myopathies. $\rho=0.82, p<0.0001$

Table 2 Correlation between 6-min walk distance and cardiopulmonary parameters using Spearman's correlation analysis

\begin{tabular}{|c|c|c|c|c|}
\hline \multirow[t]{2}{*}{ Parameters } & \multicolumn{2}{|c|}{$\begin{array}{l}\text { Healthy } \\
\text { controls }\end{array}$} & \multicolumn{2}{|c|}{$\begin{array}{l}\text { Myopathy } \\
\text { patients }\end{array}$} \\
\hline & $\rho$ & $p$ & $\rho$ & $p$ \\
\hline FVC (\% prediction) & - & - & 0.25 & 0.30 \\
\hline DLCO (\% prediction) & - & - & 0.66 & 0.007 \\
\hline $\mathrm{EF}(\%)$ & - & - & -0.10 & 0.66 \\
\hline RVSP (mmHg) & - & - & -0.28 & 0.27 \\
\hline Stroke volume $(\mathrm{mL})$, at rest & $<0.01$ & 0.99 & 0.38 & 0.07 \\
\hline Cardiac output (L), at rest & 0.10 & 0.68 & 0.33 & 0.12 \\
\hline $\begin{array}{l}\text { Stroke volume }(\mathrm{mL}) \text {, at } 6 \mathrm{~min} \text { on } \\
6 \mathrm{MWT}\end{array}$ & 0.18 & 0.48 & 0.61 & 0.002 \\
\hline $\begin{array}{l}\text { Cardiac output (L), at } 6 \text { min on } \\
6 \mathrm{MWT}\end{array}$ & 0.65 & 0.004 & 0.62 & 0.002 \\
\hline
\end{tabular}

FVC, DLCO, EF, and RVSP were not measured in healthy controls

6MWT 6-min walk test, $F V C$ forced vital capacity, DLCO diffusing capacity for carbon monoxide, $E F$ ejection fraction, $R V S P$ right ventricular systolic pressure

was not significance. A strong correlation between SV at rest and that at 6 min was observed in all subjects $(\rho=0.82$, $p<0.0001$ ) (Fig. 2).

In the healthy controls, the 6MWT distance was correlated with $\mathrm{CO}$ at $6 \mathrm{~min}$, but not with SV (Table 2). On the other hand, there was a significant correlation between 6MWT distance and DLCO $\%, \mathrm{SV}$ and $\mathrm{CO}$ at $6 \mathrm{~min}$ in the patients. There was no correlation between 6MWT distance and EF or RVSP.

\section{Discussion}

This is the first study to evaluate exercise capacity in patients with idiopathic inflammatory myopathies in relation to lung and heart function parameters. EF is a widely used parameter representing cardiac function [15], and if patients with idiopathic inflammatory myopathies have reduced $\mathrm{EF}(<60 \%)$, late gadolinium enhancement by cardiac magnetic resonance tomography is observed [5]. In this study, EF was normal in most patients and there was no correlation with 6MWT distance, whereas the SV was significantly lower than that in healthy controls. Therefore, EF was unable to be used to estimate the exercise capacity in our cohort. Pulmonary arterial hypertension would affect 6MWT distance [16], but we could not present the relationship because no patient showed high RVSP. Previously, the association of pulmonary arterial hypertension and idiopathic inflammatory myopathies has rarely been reported [17].

$\mathrm{SV}$ at $6 \mathrm{~min}$ in healthy controls was not correlated with $6 \mathrm{MWT}$ distance. It was previously reported that in healthy subjects, heart rate response contributed to $\mathrm{CO}$ or oxygen uptake rather than the SV response during exercise [18]. Additionally, varying response patterns in SV to exercise were previously observed in healthy subjects, but the mechanism was not clarified [19]. On the other hand, in this study, $\mathrm{SV}$ at rest was highly correlated with SV at $6 \mathrm{~min}$ in all subjects, and little variation in SV was noted in response to the 6MWT. Therefore, the significant deterioration in SV at rest in patients implied proportionally low SV during walking, which affected 6MWT distance. Impaired SV at rest or at 6 min suggests malfunction in the heart not detected by EF.

$\mathrm{CO}$ is known to be correlated with oxygen uptake during exercise in healthy subjects [20], and $\mathrm{CO}$ at 6 min was also correlated with 6MWT distance in healthy controls and patients. As $\mathrm{CO}$ and $\mathrm{CI}$ at 6 min were compensated for by the increased heart rate despite lower SV in patients, the difference in 6MWT distance between patients and healthy controls was unclear, which suggests that $\mathrm{CO}$ compensation during exercise influences exercise capacity.

Another variable affecting 6MWT distance was DLCO\% in patients, and this result was considered to confirm our hypothesis that accompanying interstitial lung disease affects the exercise capacity in patients. As interstitial lung disease was diagnosed in $82 \%$ (19 of 23 patients) of the patients in this study, it may be the principal manifestation affecting exercise capacity in our cohort. A significant relationship between DLCO and exercise capacity was previously confirmed in patients with idiopathic pulmonary fibrosis $[4,21]$. In addition, DLCO better represented impairment of pulmonary gas exchange than other parameters such as $\mathrm{PaO}_{2}$, alveolar-arterial oxygen pressure difference, and pulmonary capillary blood volume [22, 23]. 
FVC is another parameter on the pulmonary function test, but it was not related with 6MWT distance in the patients. For this reason, the value of FVC\%, which had a median value of $97.1 \%$, was normal compared with DLCO \%, which was $60.8 \%$. As the patients had inactive myositis, FVC may have been recovered [3] when we evaluated them. If the patients were more impaired, the exercise capacity may have been affected, as reported for patients with idiopathic pulmonary fibrosis [4].

Regarding other factors for exercise capacity, a previous study failed to find a correlation between serum creatine kinase and peak oxygen uptake or peak isometric torque in patients with inactive idiopathic inflammatory myopathies [24]. In addition, glucocorticoids and other medications may cause muscle weakness that lasts for several weeks or years [25]. Thus, many factors should be taken into consideration when evaluating the exercise capacity in patients with idiopathic inflammatory myopathies. However, we set the exclusion criteria for this study as muscle weakness in the extremities of less than 4 on manual muscle testing and leg discomfort or pain during the 6MWT to clarify the effects of cardiopulmonary functions on exercise capacity by excluding muscle factors. If all factors, such as muscle fatigue during active myositis, were included in the analysis, a different result may have been obtained.

The limitation of this study was the extraction condition of patients mentioned above. These results cannot be extended to patients who are unable to walk sufficiently. Our aim was to clarify the effects of lung and heart involvement on exercise capacity in patients with a certain condition. We demonstrated the influence of pulmonary factors in addition to cardiac factors on exercise capacity in these patients, and pulmonary rehabilitation is recommended to improve their functional status and quality of life [26]. However, idiopathic inflammatory myopathies generally involve skeletal muscle, and the relationship between muscle symptoms and exercise capacity should be examined in the future.

\section{Conclusion}

Among the manifestations of idiopathic inflammatory myopathies, we evaluated deterioration in the lung and the heart in relation to exercise capacity using the 6MWT. The SV during the 6MWT was impaired in patients compared with healthy controls, and the parameters influencing 6MWT distance were SV and $\mathrm{CO}$ at $6 \mathrm{~min}$ and DLCO\%. Interstitial lung disease may be a factor-limiting exercise capacity in patients with idiopathic inflammatory myopathies who can walk.
Acknowledgements This work was supported by JSPS KAKENHI Grant number 17K01450.

Author contributions NM performed the walking test of all patients, made the data analysis and reviewed the manuscript. FS designed research, supervised data analysis and wrote the manuscript.

\section{Compliance with ethical standards}

Conflict of interest No conflicts of interest exist.

Open Access This article is distributed under the terms of the Creative Commons Attribution 4.0 International License (http://creativeco mmons.org/licenses/by/4.0/), which permits unrestricted use, distribution, and reproduction in any medium, provided you give appropriate credit to the original author(s) and the source, provide a link to the Creative Commons license, and indicate if changes were made.

\section{References}

1. Zamora AC, Hoskote SS, Abascal-Bolado B et al (2016) Clinical features and outcomes of interstitial lung disease in anti-Jo-1 positive antisynthetase syndrome. Respir Med 118:39-45. https ://doi.org/10.1016/j.rmed.2016.07.009

2. Saketkoo LA, Ascherman DP, Cottin V, Christopher-Stine L, Danoff SK, Oddis CV (2010) Interstitial lung disease in idiopathic inflammatory myopathy. Curr Rheumatol Rev 6:108-119

3. Allenbach Y, Guiguet M, Rigolrt A et al (2015) Efficacy of rituximab in refractory inflammatory myopathies associated with antisynthetase auto-antibodies: an open-label, phase II trial. PLoS One 10:e0133702. https://doi.org/10.1371/journal.pone.0133702

4. du Bois RM, Weycker D, Albera C et al (2011) Six-minute-walk test in idiopathic pulmonary fibrosis: test validation and minimal clinically important difference. Am J Respir Crit Care Med 183:1231-1237. https://doi.org/10.1164/rccm.201007-1179OC

5. Rosenbohm A, Buckert D, Gerischer N et al (2015) Early diagnosis of cardiac involvement in idiopathic inflammatory myopathy by cardiac magnetic resonance tomography. J Neurol 262:949956. https://doi.org/10.1007/s00415-014-7623-1

6. Lundberg IE (2006) The heart in dermatomyositis and polymyositis. Rheumatology 45:iv18-iv21. https://doi.org/10.1093/rheum atology/kel311

7. Orlandi M, Barsotti S, Cioffi E et al (2016) One year in review 2016: idiopathic inflammatory myopathies. Clin Exp Rheumatol 34:966-974

8. Diederichsen LP (2017) Cardiovascular involvement in myositis. Curr Opin Rheumatol 29:598-603. https://doi.org/10.1097/ BOR.0000000000000442

9. Guerra F, Gelardi C, Capucci A, Gabrielli A, Danieli MG (2017) Subclinical cardiac dysfunction in polymyositis and dermatomyositis: a speckle-tracking case-control study. J Rheumatol 44:815-821. https://doi.org/10.3899/jrheum.161311

10. Tonelli AR, Alkukhun L, Arelli V et al (2013) Value of impedance cardiography during 6-minute walk test in pulmonary hypertension. Clin Transl Sci 6:474-480. https://doi.org/10.1111/cts.12090

11. Vasilopoulou MK, Vogiatzis I, Nasis I et al (2012) On- and off-exercise kinetics of cardiac output in response to cycling and walking in COPD patients with GOLD stages I-IV. Respir Physiol Neurobiol 181:351-358. https://doi.org/10.1016/j. resp.2012.03.014

12. Deboeck G, Taboada D, Hagan G et al (2014) Maximal cardiac output determines 6 minutes walking distance in pulmonary 
hypertension. PLoS One 9:e92324. https://doi.org/10.1371/journ al.pone.0092324

13. American Thoracic Society (2002) ATS statement: guidelines for the six-minute walk test. Am J Respir Crit Care Med 166: 111-117. https://doi.org/10.1164/rccm.166/1/111

14. Schacha L, Wigley FM, Chang B, White B, Wise RA, Gelber AC (2003) Age and risk of pulmonary arterial hypertension in scleroderma. Chest 124:2098-2104

15. Wisløff U, Støylen A, Loennechen JP et al (2007) Superior cardiovascular effect of aerobic interval training versus moderate continuous training in heart failure patients: a randomized study. Circulation 115:3086-3094. https://doi.org/10.1161/CIRCU LATIONAHA.106.675041

16. Aithala R, Alex AG, Danda D (2017) Pulmonary hypertension in connective tissue disease: an update. Int J Rheum Dis 20:5-24

17. Barsotti S, Bruni C, Cometi L et al (2017) One year in review 2017: idiopathic inflammatory myopathies. Clin Exp Rheumatol $35: 875-884$

18. Carrick-Ranson G, Hastings JL, Bhella PS et al (2013) The effect of age-related differences in body size and composition on cardiovascular determinants of $\mathrm{VO}_{2}$ max. J Gerontol A Biol Sci Med Sci 68:608-616. https://doi.org/10.1093/Gerona/gls220

19. Vella CA, Robergs RA (2005) A review of the stroke volume response to upright exercise in healthy subjects. Br J Sports Med 39:190-195. https://doi.org/10.1136/bjsm.2004.013037

20. Munch GDW, Svendsen JH, Damsgaard R, Secher NH, GonzalezAlonso J, Mortensen SP (2014) Maximal heart rate dose not limit cardiovascular capacity in healthy humans: insight from right atrial pacing during maximal exercise. J Physiol 592:377-390. https://doi.org/10.1113/jphysiol.2013.262246

21. Hallstrand TS, Boitano LJ, Johnson WC, Spada CA, Hayes JG, Raghu G (2005) The timed walk test as a measure of severity and survival in idiopathic pulmonary fibrosis. Eur Respir J 25:96-103. https://doi.org/10.1183/09031936.04.00137203

22. Wallaert B, Wemeau-Stervinou L, Salleron J, Tillie-Leblond I, Perez T (2012) Do we need exercise tests to detect gas exchange impairment in fibrotic idiopathic interstitial pneumonias? Pulm Med 2012:657180. https://doi.org/10.1155/2012/657180

23. Wémeau-Stervinou L, Perez T, Murphy C, Polge A-S, WALLAERT (2012) Lung capillary blood volume and membrane diffusion in idiopathic interstitial pneumonia. Respir Med 106:564 570. https://doi.org/10.1016/j.med.2011.12.011

24. Wiesinger GF, Quittan M, Nuhr M et al (2000) Aerobic capacity in adult dermatomyositis/polymyositis patients and healthy controls. Arch Phys Med Rehabil 81:1-5

25. Hanaoka BY, Peterson CA, Crofford LJ (2012) Glucocorticoid effects on skeletal muscle: benefit and risk in patients with autoimmune inflammatory rheumatoid diseases. Expert Rev Clin Immunol 8:695-697. https://doi.org/10.1586/ECI.12.76

26. Huppmann P, Sczepanski B, Boensch M et al (2013) Effects of inpatient pulmonary rehabilitation in patients with interstitial lung disease. Eur Respir J 42:444-453. https://doi.org/10.1183/09031 936.00081512 